\title{
Design and Prototyping of a Low-cost Portable Mechanical Ventilator
}

\author{
Kishor Jadhav', Mahendra Salunke ${ }^{2}$, Ronak Patil ${ }^{3}$, Umakant Dhande ${ }^{4}$, Saurabh S. Baviskar ${ }^{5}$ \\ 1,2,3,4 UG student, Department of Mechanical Engineering, GF's G.C.O.E., Jalgaon, India, 425001 \\ ${ }^{5}$ Assist.Prof., Department of Mechanical Engineering, GF's GF's G.C.O.E., Jalgaon, India, 425001
}

\begin{abstract}
This paper describes the design and prototyping of a low-cost portable mechanical ventilator for use in mass casualty cases and resource-poor environments. The ventilator delivers breaths by compressing a conventional bag-valve mask (BVM) with a pivoting cam arm, eliminating the need for a human operator for the BVM. An initial prototype was built out of acrylic, measuring $11.25 \times 6.7 \times 8$ inches $(285 \times 170 \times$ $200 \mathrm{~mm}$ ) and weighing $9 \mathrm{lbs}(4.1 \mathrm{~kg})$. It is driven by an electric motor powered by a 14.8 VDC battery and features an adjustable tidal volume up to a maximum of $750 \mathrm{ml}$. Tidal volume and number of breaths per minute are set via user-friendly input knobs. The prototype also features an assist-control mode and an alarm to indicate over pressurization of the system. Future iterations of the device will include a controllable inspiration to expiration time ratio, a pressure relief valve, PEEP capabilities and an LCD screen. With a prototyping cost of only $\$ 420$, the bulk-manufacturing price for the ventilator is estimated to be less than $\$ 200$. Through this prototype, the strategy of cam-actuated BVM compression is proven to be a viable option to achieve low-cost, low-power portable ventilator technology that provides essential ventilator features at a fraction of the cost of existing technology.
\end{abstract}

Keywords- Ventilator, Bag Valve Mask (BVM), LowCost, Low-Power, Portable and Automatic.

\section{I -INTRODUCTION}

V entilators are one of the most important devices to keep COVID-19 patients in the most critical condition alive. As the global demand for ventilators is increasing and there is shortage of ventilators in our country as well, also managing patients during this time is a big task, so we have designed portable rechargeable battery operated Ambu bag compressing machine, which sends real time cloud messages to the doctors and other medical authorities about the patient. We have made the prototype and we are improving it's performance by adding extra new features. It can be used for emergency purposes, in hospitals, Corona virus quarantine coaches, isolation wards and rural areas as well. The shortage of ventilators can be met effectively by developing this project. This project is a low cost yet effective ventilating system for the people affected with COVID19. These are machines which Mechanically assist patients inspire and exhale, allowing the exchange of oxygen and carbon dioxide to occur in the lungs, a process referred to as artificial respiration.

\section{II- LITERATURE REVIEW}

In [1], Designed and made a prototype of low cost portable mechanical ventilator for use in mass casually cases and resource poor environments. The ventilator 


\section{International Journal of Innovations in Engineering and Science, www.ijies.net}

compresses Bag Valve Mask (BVM) with cam arm to deliver breaths, this system eliminates human operator for compressing BVM. They developed working prototype with assist control and over pressure alarm, they system needs low power requirements and runs for 3.5 hours on single battery charge.

In [2], Developed a prototype of device to assist patients who can partially breath by their own, they made it by using minimum number of components. They used needle valve with potentiometer to replace the flow analyzer and to make it cost effective.

In [3], Determined the effects of a simple low cost oral care protocol on ventilator-associated pneumonia rates in a surgical intensive care unit, the implementation of a simple, low cost oral care protocol in surgical ICU led to a significantly decreased risk of acquiring ventilator associated pneumonia.

\section{III- METHODOLOGY}

A second prototype was built in which all the moving components were moved inside the enclosure. Enclosure dimensions were increased to accommodate the cam arm's range of motion, and to make space for the motor, microcontroller and battery pack. The enclosure's lid was made of acrylic, and hinges from the side of the unit to better constrain the top of the bag. The support ribs inside the enclosure also serve as mounting blocks for camshaft bushings. A potentiometer was coupled to the end of the shaft for use as a position feedback sensor. The degree of compression and the output air pressure can be controlled by adjusting potentiometer knob provided on the machine.

\section{Outcomes:}

1. This system is useful while shifting patients from ICU or home to ambulance or if needed at any accident site.

2. It helps persons who perform first aid by systematic easy approach, even less experienced persons can easily operate it.

3. Easy to reprogram if any changes are required in the system

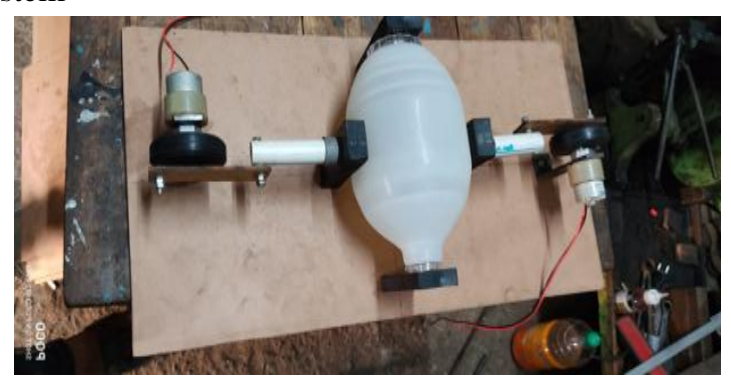

Fig. 1- Methodology

\section{User Interface:}

The three user inputs (tidal volume, bpm and I:E ratio) are set via three potentiometer knobs. Future iterations of the device will include the addition of an LCD display to show the input settings as well as airway pressure level and battery power status.

\section{Safety Features:}

To ensure that the patient is not injured, the airway pressure is monitored with a pressure sensor connected to a sensor output on the BVM. The same pressure sensor used for initiation of assist control also triggers an alarm if the pressure rises too high, alerting the physician to attend to the patient. As a further safety measure to prevent over-inflation, future iterations of the device will include a mechanical pressure relief valve.

\section{Power Delivery:}

An AC/DC converter can be used to power the ventilator directly from a wall outlet or a vehicle inverter. When external power is unavailable, the ventilator can run off of any battery capable of delivering 12-15 volt at least 3.5 Amps. For the prototype, we used a 14.8 volt, fourcell Li- Ion battery pack capable of 4.2 Amps (limited by protective circuitry), with a capacity of $2200 \mathrm{~mA}-\mathrm{hr}$.

\section{IV- ANALYSIS AND TESTING:}

\section{Battery-life Test:}

The battery life for the second prototype was tested by running the ventilator on the test lung until the battery voltage dropped to a level insufficient for operation. The device was set at maximum volume and BPM rate (30 breaths/minute). The overall duration of the test was three hours and thirty-five minutes at which point the battery was depleted. Based on the battery capacity and voltage, the electrical power consumption during this test averages to only 9 Watts (this includes the inactive time between breaths).

\section{V-CONCLUSION}

A working prototype that can be operated on a test lung has been developed. The prototype has user-controlled breath rate and tidal volume. It features assist control and an over-pressure alarm. It has low power requirements, running for 3.5 hours on one battery charge at its most demanding setting. It is portable, weighing $9 \mathrm{lbs}(4.1 \mathrm{~kg})$ and measuring 11.25 x 6.7 x 8 inches ( $285 \times 170 \times 200 \mathrm{~mm})$, and has a handle and easy to use latches. The prototype can display settings and status on a computer screen. Further development of this proof-of-concept is planned. Future iterations will 
Vol. 6, No. 10, 2021, PP. 185 -187

International Journal of Innovations in Engineering and Science, www.ijies.net

incorporate changes prompted by the results of our prototype testing. It will incorporate an adjustable inspiratory to expiratory ratio, an option missing in this prototype due to its underpowered motor. We will investigate the effects that changing the motor will cause to cost, weight and battery life.

\section{REFERENCES}

[1]. Aït-Khaled N, Enarson D, Bousquet J (2001) Chronic respiratory diseases in developing countries: the burden and strategies for prevention and management, Bulletin of the World Health Organization, 79 (10)

[2]. Chan-Yeung M, Aït-Khaled N, White N, Ip MS, Tan WC (2004) The burden and impact of COPD in Asia \& Africa. Int J Tuberc Lung Dis.

[3]. Webster's New World ${ }^{\mathrm{TM}}$ Medical Dictionary First, Second and Third Editions (May, 2008) John Wiley \& Sons, Inc.

[4]. McNeil, DG (2006) Hospitals short of ventilators if bird flu hits, NYT, May 12

[5]. Klein KR, Nagel NE (2007) Mass medical evacuation: Hurricane Katrina and nursing experiences at the New Orleans airport. DisasterManag Response. 2007 Apr-Jun;5(2):56-61

[6]. deBoisblanc, B.P. (2005) Black Hawk, please come down: reflections on a hospital's struggle to survive in the wake of Hurricane Katrina. Am J Respir Crit Care Med. Nov 15;172(10):1239-40

[7]. http://www.news-medical.net/news/2009 1020/CDCstockpiles-ventilators-for-useduring-public-healthemergency.aspx

[8]. Kerechanin CW, Cytcgusm PN, Vincent JA, Smith DG, Wenstrand DS (2004) Development of Field Portable Ventilator Systems for Domestic and Military Emergency Medical Response, John Hopkins Apl. Tech. Digest Vol25, Number 3 